\title{
Testimonio y literaturas del mundo - Notas para un debate
}

\section{Testimonio, literaturas del mundo y la perspectiva desde el Sur I}

Una de las columnas más leídas de la revista World Literature Today ${ }^{1}$ titulada "What to read now" consiste en invitar a escritores de diversas regiones del mundo para que sugieran una breve lista de lecturas, ya sea a partir de coincidencias temáticas, por compartir la misma procedencia geográfica o abordar un mismo tema social o político de actualidad, o, porque las obras seleccionadas pueden ser agrupadas bajo un mismo género literario. El número de enerofebrero de 2013 presentó cuatro recomendaciones de narrativas testimoniales dentro de las que destacan las traducciones al inglés de Biografía de un cimarrón, de Esteban Montejo y Miguel Barnet, originalmente publicada en La Habana en 1966, y Me llamo Rigoberta Menchú y así me nació la conciencia, de Menchú y Elisabeth Burgos-Debray, cuya primera edición en español data de 1983, también publicada en La Habana. ¿Cómo leer esta irrupción del género distintivo y ya canónico de la Guerra fría latinoamericana en la escena de la circulación mundial de la literatura del siglo XXI? ¿Cómo leer, en tiempos de literatura mundial, la vuelta de textos cuyas primeras ediciones de hace (casi) cincuenta y treinta años, respectivamente, marcaron el campo literario (latino) americano del mundo bipolar de la Guerra fría? ¿Se recupera acaso este género narrativo de la insurgencia y de las voces subalternas para otra literatura mundial, menos homogénea y normativizada? ¿Es posible re-leer la permanenciairrupción del testimonio revisitando y releyendo las interconexiones, dinámicas

\footnotetext{
1 World Literature Today fue fundada en 1927 con el nombre de Books Abroad por el académico Roy Temple House, director de Departamento de Lenguas Modernas de la Universidad Oklahoma. A partir de 1977 la revista cambió su nombre por el actual. Se publica bimestralmente de forma impresa y digital, conservando su objetivo inicial de fungir como un índice informativo y divulgativo de las tendencias de la literatura contemporánea a nivel mundial. Así, da a conocer tanto textos literarios como autores y una considerable cantidad de reseñas. Entre sus muchos colaboradores a lo largo de sus más de noventa años de existencia destaca el nombre de la poeta y ensayista alemana Emma Kann. Para saber más acerca de la historia de WLT, consultar la sección dedicada a ella en su página web <https://www.worldlite raturetoday.org/history >.
}

¿ Open Access. (c) 2019 Alexandra Ortiz Wallner, published by De Gruyter. (cc) BY-NC-ND This work is licensed under a Creative Commons Attribution-NonCommercial-NoDerivatives 4.0 License. https://doi.org/10.1515/9783110641134-010 
de circulación y esferas de solidaridad que emergen más allá de las asimetrías del mundo bipolar, por ejemplo, en clave de lectura Sur/South ${ }^{2}$ ?

El amplio espectro de respuestas posibles constata la complejidad discursiva inherente al fenómeno testimonial tanto como modelo narrativo precursor y configurador del tiempo revolucionario de todo un continente (Gugelberger/ Kearney 1991), como también como instancia de las "testimonial cultures" (Ahmed/Stacey 2001) que caracterizan a nuestra contemporaneidad. Así, en el marco de lo que considero su reinserción en la circulación global de la literatura (como bien simbólico y como materialidad), los debates podrían decantarse por diversos escenarios. Uno de estos se vincularía a cuestiones relativas a la colonialidad del saber (Lander/Castro-Gómez 2000), es decir, como expresión de una problemática vinculación implícita del testimonio con la noción ya fuera de uso de "Third World Literature" en las coordenadas espacio-temporales y discursivas de la "aldea global”, o, en términos de una crítica a la crítica postcolonial, tal y como Mabel Moraña escribiera, a finales de la década de 1990, en "El boom del subalterno":

\begin{abstract}
A pesar de esta salvedad, que intenta resguardar la historicidad en el proceso de asimilación y aplicación de la teorización india en otras realidades culturales, América Latina es producida, como tantas veces en su historia, como un constructo teórico legitimado desde la centralidad de discursos prestigiosos que transfieren sus categorías y agendas ideológicas a una realidad mutlifacética, cuya compleja especificidad histórica resulta inevitablemente nivelada y simplificada en el proceso de traducción teórica y negociación historiográfica. (1997: 52)
\end{abstract}

Si bien ambas posiciones, debidamente contextualizadas e historizadas, dan cuenta de debates más amplios dentro del campo de los estudios latinoamericanos y de una producción de conocimiento localizada, no dejan de configurar por ello escenarios que continúan anclados en una dinámica marcada por un pensamiento bipolar centrípeto (centro/periferia) y por su incapacidad de incorporar, en aquel momento, las histoires croisées del Sur global. Estas miradas insisten en

2 Al referir al término Sur/South lo hago en el sentido postulado en un trabajo colectivo anterior, esto es como: "[a deliberate shift in] the perspective from the often theoretically and conceptually determined cultural relationships within the Global South. Instead, it illuminates a concretely focused complex geocultural relationship, here denoted as 'SUR / SOUTH', based on examples of cultural, literary and intellectual exchanges and interrelationships between Latin America and India, in the past and present. The term 'SUR / SOUTH' thus serves as a figure of thought for a change of perspective, for a shift of focus towards the concrete framework of exchange, signified through the materiality of topics, forms of knowledge and experiences” (Klengel/Ortiz Wallner 2016: 9). 
la articulación de la diferencia, es decir, permanecen imbuidas en la matriz de las políticas de la identidad que es hora de superar.

Así, para un manejo apropiado de posiciones, teorías, opiniones y declaraciones “desde el Sur”, capaces de fracturar la verticalidad discursiva que ha caracterizado a gran parte del latinoamericanismo, es necesario que toda localización teórica vinculada a intercambios concretos entre espacios y culturas trascienda los límites de un pensamiento basado en el concepto de la diferencia. En este sentido,

[...] in order to overcome a certain epistemic blindness with regard to the positions of the Global South, a blindness diagnosed using the postcolonial criterion of "colonial difference", one also requires a precise understanding and appreciation of universal concepts such as humanity or mankind, human beings, cosmopolitanism, the world - and claims to validity, which have been formulated from different positions in the South. These concepts are not necessarily based on a mindset of contrasts, boundaries and differences. The verticality deriving from asymmetries and power structures ("North vs. South", "Centre vs. Periphery") certainly plays a role in the articulation of such concepts. But within the South-South Relationship there is also a horizontality, which leads to an articulation of similarities with relation to a third party (Europe, occidental modernity discourses), without the entire constellation of the bipolar vertical structure of "difference" necessarily taking effect. (Klengel/Ortiz Wallner 2016: 9)

Con esta primera precisión teórico-metodológica se desplaza el énfasis del fenómeno cultural del testimonio como entidad identitaria en nuestro presente hacia la relevancia de las redes de transmisión y circulación de saberes que enfatizan el cómo determinados productos culturales se reinventan y resemantizan en su paso por y existencia en distintos contextos culturales. Tomando como punto de partida la escena de lectura en World Literature Today a que me he referido antes, desarrollaré en lo que sigue el caso de circulación del fenómeno testimonial y su materialidad concreta como género narrativo desde el continente americano (específicamente desde Centroamérica) hacia el subcontinente indio, postulando que su trayectoria multirrelacional manifiesta la coexistencia de los tiempos heterogéneos de las literaturas del mundo a la vez que decanta una historia, dinámica y hasta hoy ignorada por la literatura mundial, de las redes feministas de izquierda durante la Guerra fría.

La comprensión de literatura mundial acuñada por David Damrosch (2003), esto es, como un conjunto de obras literarias que circula más allá de su contexto cultural propio, sea en su versión traducida o en su lengua original, y que en dicho proceso llega a formar parte activa e incide en un sistema literario distinto del propio (4), la leo aquí en contrapunto con la propuesta de Ottmar Ette de complejizar dicha noción en favor del término "literaturas del mundo" (2017: 37), resultantes estas de las convergencias de una serie de 
lógicas no acumulativas de una totalidad, sino acentuadoras de las carencias. ${ }^{3}$ El componente espacial de las dinámicas globales de circulación se complementa, en la noción de Ette, con una historia e historización de los movimientos y desplazamientos en los circuitos de intercambios y traslaciones. En este sentido, la verticalidad centro-periferia como lógica dominante que sigue caracterizando a las nociones clásicas de literatura mundial, es relativizada y problematizada al contraponer la pluralidad de lógicas que le son inherentes a las "literaturas del mundo", literaturas que nos recuerdan y señalan precisamente las carencias del mundo en el que existen.

El sentido operativo de una categoría como la de "literaturas del mundo" radica entonces en colocar y leer, a contrapelo, en forma de red no jerárquica ni normativa, sino en razón de su movilidad, mutabilidad, variación y fuga, los disensos, diferencias y multiplicidades de las producciones literarias insertas en circuitos de circulación concretos. Esto implica, para el caso del lugar de enunciación que denominamos “América Latina”, pensar la historia de la circulación de saberes como una trayectoria de idas y de vueltas. El caso del testimonio, tal y como lo propongo comprender y releer aquí en su circulación Sur-Sur (específicamente de Centroamérica hacia la India), es la historia de una trayectoria productiva de transferencias literarias e intelectuales entre culturas testimoniales, estudios subalternos y feminismos del Sur.

\section{Testimonio, literaturas del mundo y la perspectiva desde el Sur II}

Con el auge de los debates acerca de la literatura mundial y las literaturas del mundo se fue recuperando, a inicios del siglo XXI, un espacio de enunciación para las teorías y los estudios literarios comparados. Paralelamente se abría el espacio del debate a partir de las voces provenientes de lugares de enunciación otros, ejemplarmente reunidos bajo categorías como la del Sur global, Sur/

3 "El concepto de las literaturas del mundo, por el contrario, no presupone ninguna abundancia tal. Su forma de pensar multilógica siempre tiene presente que numerosas otras lógicas todavía no están incluidas en él e incluso que estas lógicas desconocidas, no exploradas, les faltan, por naturaleza, al discurso propio y a la concepción propia de las literaturas del mundo. Así, el discurso de las literaturas del mundo no cae en la trampa de la abundancia, sino que parte de una carencia, de una escases, de una privación que no se puede encubrir con ningún tipo de acumulación cuantitativa, ya que las literaturas del mundo no se dejan encasillar ni delimitar espacialmente y muestran siempre brechas y lagunas" (Ette 2017: 66; traducción mía). 
South o relaciones Sur-Sur (véase Mann/Phaf-Rheinberger 2014; Klengel/Ortiz Wallner 2016; Mahler 2017). Estas categorías comparten una problematización sistemática de la perspectiva eurocéntrica que había marcado los estudios y nociones de la globalización vigentes (acuñados, por ejemplo, en el término "Tercer mundo"), desplazando su atención crítica a las histoires croisées más amplias y complejas, entre Asia, África y América Latina. Es en este corte en el que cobra aún más sentido la comprensión del género testimonial como un "lenguaje global" 4 transversal capaz de situarse y operar bajo las coordenadas de más de un sistema literario y en diversos continentes, no necesariamente de forma simultánea sino más bien en múltiples y heterogéneos tiempos. Así, su trayectoria Sur/South como travelling concept incluiría una serie de intervenciones en planos diferenciados como el de una cultura global vinculada a los reclamos de justicia y políticas de la memoria, hasta su circulación exitosa como texto icónico de la literatura latinoamericana en el mercado global del libro como consta, por ejemplo, para el caso de la traducción al inglés del texto de Menchú anunciado en el sitio de la no menos icónica Verso Books como un "global bestseller". ${ }^{5}$

Kavita Panjabi, especialista en literatura comparada y reconocida académica feminista, trazó ya a inicios de la década de 1990, desde la India, el trayecto Sur/ South del testimonio: "One of the first cases (perhaps the first case) of Latin American influence on Indian Literature in the context of women's writing is that of Sandino's Daughters [...] on We Were Making History: Life Stories of Women in the Telengana People’s Struggle”6 (1992: 58s.) y más adelante continúa:

4 Para un panorama reciente sobre el testimonio como fenómeno cultural en un contexto ampliado mundial ver la introducción "Perspektiven auf ein kulturelles Phänomen: Zeugenschaft in der Romania” (Nickel/Ortiz Wallner 2014: 7-15).

5 Véase el anuncio en la página de Verso: https://www.versobooks.com/books/445-irigoberta-menchu.

6 "We Were Making History: Women and the Telengana Uprising, a collection of testimonies of women who participated in the Telengana People's Struggle of the forties in the state of Hyderabad, recorded and edited by the Stree Shakti Sangathana, was the first widely read collection of women's testimonial literature in India and was published in 1989. [...] The editors of the testimonios acknowledge this influence thus: 'When we first chose to do this study, one of our own aims was to recover our own history (we saw the women in the Telengana Struggle as founders of a history of women's action in Andhra, indeed in India itself). So we thought we would be tracing a lineage. But after we had done nearly forty interviews, we decided that it would be best to publish them as life stories. We now thought of it as a book that was theirs, as much as it was ours. We were also encouraged because we had read and enjoyed [...] Sandino’s Daughters (280)'” (Panjabi 1992: 58s.). 
This instance reflects the direct influence of Central American women's testimonies on Indian feminist historiography, as a precedent and persuasive force, encouraging the creation of space for a new and important genre. [...] Given the strong parallels in the political and historical experiences of women in these cases, there was ready ground here for the reception of this influence; the Telengana women's narratives, like the Central American testimonios [...] are situated in the historical context of crises in nationalism in contemporary postcolonial societies, and the increasing articulation of the role of women in these struggles. (Panjabi 1992: 59)

Aquel primer momento de contacto y recepción, Panjabi, así como el colectivo feminista de izquierda Stree Shakti Sanghatana que editó We Were Making History, lo ubican claramente en el trabajo doble (académico y de activismo político) de reivindicación de la historia de las mujeres como un acto público y privado de intervención política. Así, el colectivo de editoras que recopiló los testimonios y dio forma a We Were Making History (1989) escribía en su introducción programática: "Women's history, then, is an intervention: its intent is more political than archival... As we search out and record the histories in this book, we set out to reclaim a past and celebrate a lineage of resistance and growth, for to be deprived of a past is to inherit an impoverished present and a future sealed off from change" (Sanghatana 1989: 19).

La temprana lectura y recepción del clásico de Margaret Randall Sandino's Daughters (1981), así como de los testimonios de Domitila y Rigoberta Menchú (véase Panjabi 1992 y 2009a) abrieron una serie de debates en los circuitos de la cultura letrada de las izquierdas en la India. El testimonio no fue visto como un sustituto de la historiografía, sino como un género autónomo capaz de ocupar un lugar que haría que las voces silenciadas se escuchasen de forma diferenciada y no naturalizada dado que, simultáneamente, se trataba de un género "nuevo", uno que apenas empezaba a ser nombrado en India en 1989, año tan significativo para la historia global. Es precisamente esta intersección entre una emergente forma de literatura insurgente en India y la circulación de la misma en círculos letrados de diferentes continentes, es decir entre Centro-/Latinoamérica y la India, que emerge una trayectoria Sur/South hasta hoy desatendida e ignorada por la teoría y crítica latinoamericanas.

Se amplía así el horizonte de comprensión del fenómeno testimonial dado que en su trayectoria del Sur al Sur visibiliza una serie de aspectos que le adjudican un papel relevante en las literaturas del mundo y en las historias literarias en que surge, ya no comprendidas estas como manifestaciones de una comunidad imaginada restringida y restrictiva, sino como historias literarias conectadas entre sí. Por un lado, las reflexiones de que es objeto desde India muestran la historia de sus vinculaciones con géneros "anteriores" como la novela y las narraciones autobiográficas, de origen occidental y no occidental: "testimonio did 
not develop from a vacuum, it belongs to and is a development from traditions of political literary texts that prevailed in both the Indian and the Latin American contexts" (Panjabi 1992: 60). Recurriendo a la tradición no occidental de las narraciones autobiográficas en India y tomando como caso paradigmático la autobiografía de Gandhi publicada en 1948 en su traducción al inglés, esto es, al inicio de la India independiente, Panjabi (1992; 2009) reconstruye a partir de ese primer momento postcolonial una serie de entrelazamientos de las dimensiones retórica, metafórica y política presentes posteriormente en el género del testimonio una vez que ha entrado (a finales de la década de 1980) vía los testimonios centroamericanos en el sistema literario indio.

Una de las claves de lectura radica precisamente en poner el énfasis en el cómo son analizadas las genealogías de producción y recepción de textos cuando estos se mueven fuera de "su" lugar, esto es, fuera de su aparente lugar natural de origen. Así, Panjabi va perfilando en sus estudios pioneros (1992; 2009a) las rutas de circulación y recepción y las transformaciones estético-políticas del género del testimonio en India proponiendo una periodización literaria a partir de la publicación de We Were Making History hasta "Living to tell their tale: Testimonio as subaltern voice in India and Latin America”, un seminario organizado en el 2007 en Nueva Delhi en el Center for Spanish and Latin American Studies de la universidad Jamia Milia Islamia, constatando en dicho recorrido una ampliación que incluiría ya no únicamente las luchas de las mujeres militantes de izquierda por contar con su propia historia, sino también como género idóneo para narrar las historia de vida de los dalits. ${ }^{7}$ Un caso ejemplar de las interconexiones entre feminismos, historias de vida de los dalits ${ }^{8}$ y testimonio se halla en la compilación de Sharmila Rege Writing Caste/Writing Gender:

7 La hoy llamada literatura dalit ("Dalit Literature”) es un género ya consagrado en India y en otros contextos. En un artículo publicado en el 2015 en The Times of India titulado "Dalit literature goes global” Martand Kaushik resume el estado de cuestión así: “"The circulation of Dalit literature [...] is important to deconstruct an idea of India that is pervasive, and one that many diasporic Indians seek to cultivate: India as non-violent, Hinduism as mythological, anti-orthodoxy and benevolent, and both as peace-loving,' says Toral Gajarawala, an associate professor at NYU. 'The knowledge of India that circulates in the West is caste-free. Dalit studies offer a corrective to this 'idea of India' in an important way'” (Kaushik 2015).

8 Acerca del papel de la literatura dalit como un género emergente, Panjabi observa: "Dalit autobiographies, which have been compared to African-American slave narratives, are now also being read in the critical framework of the Latin American testimonio in terms of their narrative strategies and content, and their institutional location and function vis- $a$-vis critical academic discourse. [. . . Even though the writings by Dalit women appeared bizarre and illogical to many readers and critics in the non-Dalit literary circles, today these testimonios have become crucial to think and theorize about the literary and social spheres" (2009b: 12). 
Narrating Dalit Women's Testimonios (2006), libro con el cual el testimonio - asumido ya sin traducción al inglés como lo demuestra el título- terminaría por constatar la disrupción del discurso tradicional de la historia literaria india como manifestación de una historia literaria mucho más compleja y global, marcada por la trayectoria Sur/South de un género narrativo de los márgenes americanos hacia los márgenes de la sociedad hiperestratificada de la India:

\begin{abstract}
Another dimension to this debate also included the view that the original name, testimonio, be preserved, for if we could ultimately retain the Anglo-American term "novel" in India, does the reluctance to take on the Latin American testimonio not reflect a bias against acknowledging the literary interaction with Latin American influence? [...] The point is thus not about the western or Latin American or Indian source of nomenclature but about the consensus on the nature of the conventions of the genre in a specific culture, and the horizons of expectation a particular generic naming may set up. For a genre frames the reader's interpretation of a text; and the specific conventions of the genre, draw readers into identifying the significant features of the genre, as distinct from others, operative in the text. (Panjabi 2009b: 9s.)
\end{abstract}

Ahora bien, no se trata en esta historia literaria de las relaciones Sur-Sur de borrar las complejidades y paradojas de cada sistema literario o de ignorar los contextos histórico-culturales específicos en que el género testimonial surge e interactúa. ${ }^{9}$ Lo que me interesa destacar en toda esta reflexión es la potencialidad de una lectura a contrapelo que la perspectiva teórico-metodológica situada del concepto Sur/South devuelve a los estudios literarios. Al cuestionar el archivo y el canon dominantes, aún de un género literario menor como lo es el testimonio, y al rastrear su historia alternativa de circulación fuera de la territorialidad americana, nos ubicaremos en un espacio de enunciación más complejo y, a la vez, menos normativo capaz de releer las redes de solidaridad literaria de los feminismos del Sur hacia finales de la Guerra fría. Solidaridades literarias que, desde la historia de la circulación del testimonio en clave Sur/ South, develan renovadas relaciones entre estética y política. El testimonio, en esta particular constelación de relaciones y transferencias culturales puede y debe ser, una vez más, releído en toda su complejidad como parte de las literaturas del mundo.

9 Rege apunta acertadamente a una de las particularidades del testimonio en India cuando afirma: "In order to bring 'new insights and theories into elite brahmanical institutions of academia' as well as to integrate Dalits into mainstream society, it is imperative that the testimonios of Dalits should be regarded as a medium of 'resistance and organized anti-caste struggle"” (2006: 15). 


\section{Bibliografía}

Ahmed, Sara/Stacey, Jackie (2001): “Testimonial Cultures: An Introduction”. En: Cultural Values, 5, 1, pp. 1-6.

Alexander, Jacqui M./Mohanty, Chandra Talpade (eds.) (1997): Feminist Genealogies, Colonial Legacies, Democratic Futures. Londres/Nueva York: Routledge.

Damrosch, David (2003): What is World Literature? Princeton: Princeton University Press.

Ette, Ottmar (2017): WeltFraktale. Wege durch die Literaturen der Welt. Stuttgart: Metzler.

Gandhi, M.K. (1948): The Story of My Experiments With Truth. Trad. Mahadev Desai.

Washington D. C.: Public Affairs Press.

Gugelberger, Georg/Kearney, Michael (eds.) (1991): "Voices for the Voiceless: Testimonial Literature in Latin America”. En: Latin American Perspectives, 18 (Special Issue), pp. 3-14.

Gugelberger, Georg M. (ed.) (1996): The Real Thing: Testimonial Discourse and Latin America. Durham: Duke University Press.

Hecht, Tobias (2013): “What to Read Now: Testimonial Narratives”. En: WLT, enero-febrero 2013, <https://www.worldliteraturetoday.org/2013/january/what-read-now-testimonialnarratives> (última visita: 31/05/2018).

Jameson, Fredric (1986): “Third World Literature in the Era of Multinational Capitalism”. En: Social Text, 15, pp. 65-88.

Kannabiran, Vasantha (1986): “Report from SSS. A Women's Group in Hyderabad, Andra Pradesh, India”. En: Feminist Studies, 12, 3, pp. 601-612.

Kaushik, Martand (2015): “Dalit literature goes global”. En: The Times of India, 05/04/2015, <https://timesofindia.indiatimes.com/home/sunday-times/deep-focus/Dalit-literaturegoes-global/articleshow/46810541.cms> (última visita: 31/05/2018).

Klengel, Susanne/Ortiz Wallner, Alexandra (eds.) (2016): Sur /South: Poetics and Politics of Thinking Latin America /India. Fráncort del Meno/Madrid: Vervuert/Iberoamericana.

Lander, Edgardo/Castro-Gómez, Santiago (eds.) (2000): La colonialidad del saber: Eurocentrismo y Ciencias Sociales. Buenos Aires: CLACSO.

Mahler, Anne Garland (2017): “Global South”. En: O’Brien, Eugene (ed.): Bibliographies in Literary and Critical Theory. Oxford/Nueva York: Oxford University Press, <http://www.ox fordbibliographies.com/view/document/obo-9780190221911/obo-9780190221911-0055. xml?rskey=eMzKAW\&result=16> (última visita: 31/05/2018). pp. 1-2, 14-15.

Mann, Michael/Phaf-Rheinberger, Ineke (eds.) (2014): Beyond the Line. Cultural narratives of the Southern Oceans. Berlín: Neofelis.

Menchú, Rigoberta/Burgos-Debray, Elisabeth (1983): Me llamo Rigoberta Menchú y así me nació la conciencia. La Habana: Casa de las Américas.

Menchú, Rigoberta/Burgos-Debray, Elisabeth (1984): I, Rigoberta Menchu: An Indian Woman in Guatemala. Ed. y introd. de Elisabeth Burgos-Debray. Trad. Ann Wright. Londres: Verso.

Montejo, Esteban/Barnet, Miguel (1966): Biografía de un cimarrón. La Habana: Instituto de Etnología y Folclor.

Moraña, Mabel (1997): “El boom del subalterno”. En: Revista de Crítica Cultural, 15, pp. 48-53.

Nayar, Pramod K. (2012): “The Poetics of Postcolonial Atrocity: Dalit Life Writing, Testimonio, and Human Rights”. En: Ariel: A Review of International English Literature, 42, 3-4, pp. 237-264. 
Nickel, Claudia/Ortiz Wallner, Alexandra (eds.) (2014): Zeugenschaft. Perspektiven auf ein kulturelles Phänomen. Heidelberg: Winter.

Panjabi, Kavita (ed.) (2009a): Jadavpur Journal of Comparative Literature, 46 (Special Issue: "Dalit Narratives and the Testimonial Genre in India").

Panjabi, Kavita (ed.) (2009b): "The Testimonio in Latin America and India: Critical Contestations of the Collective Voice”. En: Jadavpur Journal of Comparative Literature, 46, pp. 7-24.

Panjabi, Kavita (ed.) (2004): “Transcultural Politics and Aesthetics: Testimonial Literature". En: Literary Studies in India. Kolkata: D.S.A./Comparative Literature Jadavpur University, pp. 122-143.

Panjabi, Kavita (ed.) (1992): “The Generic Location of Women's Testimonial Literature and its Social Function in India and Latin America”. En: Jadavpur Journal of Comparative Literature, 30, pp. 58-65.

Rabasa, José/Sanjinés, Javier/Carr, Robert (eds.) (1994): Dispositio/n, 19, 46 (Special Issue: "Subaltern Studies in the Americas").

Randall, Margaret (1981): Sandino's Daughters: Testimonies of Nicaraguan Women in Struggle. Vancouver: New Star Books.

Rege, Sharmila S. (2006): Writing Caste/Writing Gender: Narrating Dalit Women's Testimonios. Nueva Dehli: Zubaan.

Sklodowska, Elzbieta (1992): Testimonio hispanoamericano: Historia, teoría, poética. Nueva York: Peter Lang.

Sanghatana, Stree Shakti (1989): We Were Making History: Life Stories of Women in the Telengana People's Struggle. Nueva Delhi: Kali for Women. 
